One- el ect r on di at om cs i n moment um space. I . I mpl i cat i on of first iterated sol uti ons for the ground state

\begin{tabular}{|c|c|}
\hline 著者 & KOGA Toshi kat su \\
\hline $\begin{array}{l}\text { jour nal or } \\
\text { publ i cat } i \text { on } t i t l e\end{array}$ & The $\mathrm{j}$ our nal of chemi cal physi cs \\
\hline vol une & 83 \\
\hline number & 5 \\
\hline page $r$ ange & $2328-2333$ \\
\hline year & 1985-09-01 \\
\hline URL & ht t p: //hdl . handl e. net /10258/938 \\
\hline
\end{tabular}




\title{
One-electron diatomics in momentum space. I. Implication of first iterated solutions for the ground state
}

\author{
Toshikatsu Koga \\ Department of Applied Chemistry and Department of Applied Science for Energy, Muroran Institute \\ of Technology, Muroran, Hokkaido, 050 Japan
}

(Received 29 March 1985; accepted 29 May 1985)

\begin{abstract}
For one-electron diatomic systems, an iterative solution of the momentum-space Schrödinger equation is examined using the Fock transformation which enables us to expand the kernel of the integral equation by the four-dimensional spherical harmonics. Starting from the united atom (UA) and simple LCAO approximations, first iterated solutions are derived and their properties are analyzed. The corresponding approximate energy eigenvalues are also obtained as a function of the internuclear distance $R$. The result from the LCAO starting function is found to be reliable semiquantitatively: in the range of $0 \leqslant R \leqslant 20$, the maximum errors of the ground-state electronic energy are $4.7 \%$ and $1.7 \%$, respectively, for the $\mathrm{H}_{2}^{+}$and $\mathrm{HeH}^{2+}$ systems, when compared with the exact values.
\end{abstract}

\section{INTRODUCTION}

In recent papers, ${ }^{1,2}$ momentum-space perturbation equations for the $\mathrm{H}(1 s)-\mathrm{H}^{+}$and $\mathrm{H}(1 s)-\mathrm{H}(1 s)$ long-range interactions have been exactly solved in their Schrödinger forms. The approach used there for the solution of integral equations in momentum space consists of two steps: the momentum vector $\mathbf{p}$ of an electron is first transformed from the three-dimensional hyperplane onto the four-dimensional hypersphere (Fock transformation). ${ }^{3-7}$ Then the kernel of the integral equation is expanded by the four-dimensional spherical harmonics $\left\{Y_{n l m}\right\}$. Due to the orthonormal property of $\left\{\boldsymbol{Y}_{n l m}\right\}$, the integral equation is finally reduced to a linear equation which exactly determines the perturbation wave function.

In the present paper, we apply the same method to oneelectron diatomic systems such as $\mathrm{H}_{2}^{+}$and $\mathrm{HeH}^{2+}$ in their ground states and examine an iterative solution. The momentum-space solution of the $\mathrm{H}_{2}^{+}$. molecule was first investigated by McWeeny. ${ }^{8} \mathrm{He}$ concluded that the iterative method is not very convenient because the required integrations are quite complicated. Introducing the method of Fock transformation, however, Shibuya and Wulfman ${ }^{4}$ showed that these integrations are possible even when one starts from the LCAO approximation for the iteration, and estimated the ground-state electronic energy of $\mathrm{H}_{2}^{+}$system at a small internuclear distance $R$. We therefore proceed with our study along the line developed by Shibuya and Wulfman, but we devise a new method to determine approximate wave functions and eigenvalues for a wide range of $R$. We also analyze the property of first iterated solutions in detail, which was not given in the work of Shibuya and Wulfman. In the next section, we outline the Fock transformation and define the four-dimensional spherical harmonics $\left\{Y_{n l m}\right\}$. In addition, two functions $S_{n^{\prime} l^{\prime} m^{\prime}}^{n l m}(\mathbf{R})$ and $W_{n^{\prime} l^{\prime} m^{\prime}}^{n l m}(\mathbf{R})$ are discussed, which are useful for the many-center problem in Fock space. Though these functions were originally introduced by Shibuya and Wulfman, ${ }^{4}$ we here clarify various important properties and relations. In Sec. III, first iterated solutions are derived starting from the united atom (UA) and LCAO wave functions and their implication is discussed. Asymptotic behaviors of the solutions are also examined for small and large internuclear distances. The numerical results for the $\mathrm{H}_{2}^{+}$ and $\mathrm{HeH}^{2+}$, together with the analysis of the asymptotic behavior, show that the iterative solution for one-electron diatomics is promising particularly when one adopts the LCAO starting function. Atomic units are used throughout this paper.

\section{FOUR-DIMENSIONAL SPHERICAL HARMONICS AND RELATED FUNCTIONS}

\section{A. Four-dimensional spherical harmonics $Y_{n / m}(\Omega)$.}

We project the three-dimensional momentum space onto the four-dimensional sphere centered at the momentum space origin with the radius $p_{0}$. As we will see later, the value of $p_{0}$ is directly related to the energy eigenvalue of the system under consideration and is determined at the final stage of the solution. The projective origin is taken at the point $\left(0,0,0,-p_{0}\right)$ in the four-dimensional (Fock) space. Then a momentum vector $\mathbf{p}[=(p, \theta, \phi)$ in the polar coordinates] in the hyperplane is transformed to a point on the hypersphere, which is specified by the three angles $(\alpha, \theta, \phi)$. The two angles $\theta$ and $\phi$ have usual meaning, while the new angle $\alpha(0 \leqslant \alpha<\pi)$ represents the radius $p(0 \leqslant p<\infty)$. The transformation is ${ }^{3-7}$

$$
\begin{aligned}
& p=p_{0} \tan (\alpha / 2), \\
& d \mathbf{p}=\left[\left(p^{2}+p_{0}^{2}\right) / 2 p_{0}\right]^{3} d \Omega, \\
& d \Omega=\sin ^{2} \alpha \sin \theta d \alpha d \theta d \phi,
\end{aligned}
$$

where $\Omega$ means the collection of the angles $\alpha, \theta$, and $\phi$. In Eq. (1b) and hereafter, we conveniently continue using $p$ to represent $p_{0} \tan (\alpha / 2)$ even after the Fock transformation is carried out.

In Fock space, we can expand $\left|\mathbf{p}-\mathbf{p}^{\prime}\right|^{-2}$ (which appears as a kernel of the momentum-space Schrödinger equation) as $^{3-5}$

$$
\left|\mathbf{p}-\mathbf{p}^{\prime}\right|^{-2}=4 p_{0}^{2}\left[4 \sin ^{2}(\omega / 2)\left(p^{2}+p_{0}^{2}\right)\left(p^{\prime 2}+p_{0}^{2}\right)\right]^{-1},
$$




$$
\left[4 \sin ^{2}(\omega / 2)\right]^{-1}=2 \pi^{2} \sum_{n l m} n^{-1} Y_{n l m}^{*}\left(\Omega^{\prime}\right) Y_{n l m}(\Omega),
$$

where $\omega$ is the angle spanned between the two points $\Omega(=\mathbf{p})$ and $\Omega^{\prime}\left(=\mathbf{p}^{\prime}\right)$. $\left\{Y_{n l m}(\Omega)\right\}$ are four-dimensional spherical harmonics defined by

$$
\begin{aligned}
Y_{n l m}(\Omega)= & (-i)^{l} C_{n, l}(\alpha) Y_{l m}(\theta, \phi), \\
C_{n, l}(\alpha)= & \{2 n(n-l-1) ! /[\pi(n+l) !]\}^{1 / 2} \sin ^{l} \alpha \\
& \times\left[d^{l} C_{n-1}^{1}(\mu) / d \mu^{l}\right]_{\mu=\cos \alpha},
\end{aligned}
$$

where $\left\{Y_{l m}(\theta, \phi)\right\}$ are usual three-dimensional spherical harmonics and $C_{n}^{v}(\mu)$ denotes the Gegenbauer polynomial. The $\left\{Y_{n l m}\right\}$ satisfy

$$
\begin{aligned}
& \int Y_{n l m}^{*}(\Omega) Y_{n^{\prime} l^{\prime} m^{\prime}}(\Omega) d \Omega=\delta_{n n^{\prime}} \delta_{l l^{\prime}} \delta_{m m^{\prime}}, \\
& Y_{n l m}^{*}(\Omega)=(-1)^{l-m} Y_{n l-m}(\Omega) .
\end{aligned}
$$

The momentum-space hydrogenic wave function $\Phi_{n l m}$ is expressed as

$$
\Phi_{n l m}=4 p_{0}^{5 / 2}\left(p^{2}+p_{0}^{2}\right)^{-2} Y_{n l m}(\Omega)
$$

where $p_{0}$ plays a role of the exponent $Z / n$, and the integers $n$, $l, m$ the three quantum numbers.

\section{B. Functions $S_{n^{\prime} / m^{\prime}}^{n / m}(\mathbf{R})$ and $W_{n^{\prime} / m^{\prime}}^{n / m}(\mathbf{R})$}

Let us define a function $S_{n^{\prime} l^{\prime} m^{\prime}}^{n l m}(\mathbf{R})$ by

$$
S_{n^{\prime} l^{\prime} m^{\prime}}^{n l m}(\mathbf{R})=\int \exp (i \mathbf{p} \cdot \mathbf{R}) Y_{n l m}(\Omega) Y_{n^{\prime} l^{\prime} m^{\prime}}^{*}(\Omega) d \Omega .
$$

Then we see ${ }^{4}$

$$
\begin{aligned}
& {\left[S_{n^{\prime} l^{\prime} m^{\prime}}^{n l m}(\mathbf{R})\right]^{*}=S_{n l m}^{n^{\prime} l^{\prime} m^{\prime}}(-\mathbf{R}),} \\
& S_{n^{\prime} l^{\prime} m^{\prime}}^{n l(0)}=\delta_{n n^{\prime}} \delta_{l l^{\prime}} \delta_{m m^{\prime}},
\end{aligned}
$$

and

$$
\begin{aligned}
& \exp (+i \mathbf{p} \cdot \mathbf{R}) Y_{n l m}(\Omega)=\sum_{n^{\prime} l^{\prime} m^{\prime}} S_{n^{\prime} l^{\prime} m^{\prime}}^{n l m}(\mathbf{R}) Y_{n^{\prime} l^{\prime} m^{\prime}}(\Omega), \\
& \exp (-i \mathbf{p} \cdot \mathbf{R}) Y_{n l m}(\Omega)=\sum_{n^{\prime} l^{\prime} m^{\prime}}\left[S_{n l m}^{n^{\prime} l^{\prime} m^{\prime}}(\mathbf{R})\right]^{*} Y_{n^{\prime} l^{\prime} m^{\prime}}(\Omega) .
\end{aligned}
$$

Using the abbreviation $N=(n l m)$, we find from Eq. (6a) that $\exp \left[i \mathbf{p} \cdot\left(\mathbf{R}_{1}+\mathbf{R}_{2}\right)\right] Y_{n l m}(\Omega)$ can be expanded in two different ways:

$$
\begin{aligned}
& \exp \left[i \mathbf{p} \cdot\left(\mathbf{R}_{1}+\mathbf{R}_{2}\right)\right] Y_{N}(\Omega) \\
& =\left\{\begin{array}{l}
\sum_{N^{\prime}} S_{N^{\prime}}^{N}\left(\mathbf{R}_{1}+\mathbf{R}_{2}\right) Y_{N^{\prime}}(\Omega), \\
\sum_{N^{\prime}} \sum_{N^{\prime \prime}} S_{N^{\prime}}^{N}\left(\mathbf{R}_{1}\right) S_{N^{\prime \prime}}^{N^{\prime}}\left(\mathbf{R}_{2}\right) Y_{N^{\prime \prime}}(\Omega) .
\end{array}\right.
\end{aligned}
$$

Comparison of the right-hand sides of Eq. (7a) gives a sum rule

$S_{N^{\prime}}^{N}\left(\mathbf{R}_{1}+\mathbf{R}_{2}\right)=\sum_{N^{\prime \prime}} S_{N^{\prime \prime}}^{N}\left(\mathbf{R}_{1}\right) S_{N^{\prime}}^{N^{\prime \prime}}\left(\mathbf{R}_{2}\right)=\sum_{N^{\prime \prime}} S_{N^{\prime}}^{N^{\prime \prime}}\left(\mathbf{R}_{1}\right) S_{N^{\prime \prime}}^{N}\left(\mathbf{R}_{2}\right)$

which can be generalized to
$S_{N_{j}}^{N}\left(\mathbf{R}_{1}+\cdots+\mathbf{R}_{j}\right)=\sum_{N_{1}} \cdots \sum_{N_{j-1}} S_{N_{1}}^{N}\left(\mathbf{R}_{1}\right) S_{N_{2}}^{N_{1}}\left(\mathbf{R}_{2}\right) \cdots S_{N_{j}}^{N_{j-1}}\left(\mathbf{R}_{j}\right)$

if we consider different expansions of $\exp \left[i \mathbf{p} \cdot\left(\mathbf{R}_{1}+\cdots+\mathbf{R}_{j}\right)\right] Y_{N}(\Omega)$.

Integrating the product of Eq. (6a) and its complex conjugate over $\Omega$, we have

$$
\sum_{v \lambda \mu} S_{v \lambda \mu}^{n l m}(\mathbf{R})\left[S_{v \lambda \mu}^{n^{\prime} l^{\prime} m^{\prime}}(\mathbf{R})\right]^{*}=\delta_{n n^{\prime}} \delta_{l l^{\prime}} \delta_{m m^{\prime}},
$$

and especially for $(n l m)=\left(n^{\prime} l^{\prime} m^{\prime}\right)$,

$$
\sum_{n^{\prime} l^{\prime} m^{\prime}}\left|S_{n^{\prime} l^{\prime} m^{\prime}}^{n l m}(\mathbf{R})\right|^{2}=1,
$$

from which it follows that

$$
0 \leqslant\left|S_{n^{\prime} l^{\prime} m^{\prime}}^{n l m}(\mathbf{R})\right| \leqslant 1 .
$$

A position-space picture of the function $S_{n^{\prime} l^{\prime} m^{\prime}}^{n l m}(\mathbf{R})$ is given in the Appendix.

We next define a function $W_{n^{\prime} l^{\prime} m^{\prime}}^{n l m}(\mathbf{R})$ as

$$
\begin{aligned}
W_{n^{\prime} l^{\prime} m^{\prime}}^{n l m}(\mathbf{R})= & \sum_{v \lambda \mu} v^{-1} S_{v \lambda \mu}^{n l m}(\mathbf{R}) \int Y_{v \lambda \mu}(\Omega) \\
& \times\left[\exp (i \mathbf{p} \cdot \mathbf{R}) Y_{n^{\prime} l^{\prime} m^{\prime}}(\Omega)\right]^{*} d \Omega \\
= & \sum_{v \lambda \mu} v^{-1} S_{v \lambda \mu}^{n l m}(\mathbf{R})\left[S_{v \lambda \mu}^{n^{\prime} l^{\prime} m^{\prime}}(\mathbf{R})\right]^{*}
\end{aligned}
$$

Then the following relations hold:

$$
\begin{aligned}
& {\left[W_{n^{\prime} l^{\prime} m^{\prime}}^{n l m}(\mathbf{R})\right]^{*}=W_{n l m}^{n^{\prime} l^{\prime} m^{\prime}}(\mathbf{R}),} \\
& W_{n^{\prime} l^{\prime} m^{\prime}}^{n l m}(\mathbf{0})=n^{-1} \delta_{n n^{\prime}} \delta_{l l}, \delta_{m m^{\prime}}, \\
& \sum_{n^{\prime} l^{\prime} m^{\prime}} n^{\prime-1} S_{n^{\prime} l^{\prime} m^{\prime}}^{n l m}(\mathbf{R}) Y_{n^{\prime} l^{\prime} m^{\prime}}(\Omega) \\
& \quad=\sum_{n^{\prime} l^{\prime} m^{\prime}} W_{n^{\prime} l^{\prime} m^{\prime}}^{n l m}(\mathbf{R})\left[\exp (i \mathbf{p} \cdot \mathbf{R}) Y_{n^{\prime} l^{\prime} m^{\prime}}(\Omega)\right] .
\end{aligned}
$$

Similar to the function $S_{n^{\prime} l^{\prime} m^{\prime}}^{n l m}(\mathbf{R})$, we can derive several sum rules for $W_{n^{\prime} l^{\prime} m^{\prime}}^{n l m}(\mathbf{R})$. For example,

$\sum_{n^{\prime} l^{\prime} m^{\prime}} W_{n^{\prime} l^{\prime} m^{\prime}}^{n l m}(\mathbf{R}) S_{n l m}^{n^{\prime} l^{\prime} m^{\prime}}(\mathbf{R})=n^{-1} S_{n l m}^{n l m}(\mathbf{R})$

$\sum_{v \lambda \mu} v^{-2} S_{v \lambda \mu}^{n l m}(\mathbf{R})\left[S_{v \lambda \mu}^{n^{\prime} l^{\prime} m^{\prime}}(\mathbf{R})\right]^{*}=\sum_{v \lambda \mu} W_{\nu \lambda \mu}^{n l m}(\mathbf{R})\left[W_{v \lambda \mu}^{n^{\prime} l^{\prime} m^{\prime}}(\mathbf{R})\right]^{*}$

and a special case of Eq. $(11 \mathrm{~b})$ for $(n l m)=\left(n^{\prime} l^{\prime} m^{\prime}\right)$ is

$$
\begin{aligned}
\sum_{n^{\prime} l^{\prime} m^{\prime}}\left|W_{n^{\prime} l^{\prime} m^{\prime}}^{n l m}(\mathbf{R})\right|^{2} & =\sum_{n^{\prime} l^{\prime} m^{\prime}} n^{\prime-2}\left|S_{n^{\prime} l^{\prime} m^{\prime}}^{n l m}(\mathbf{R})\right|^{2} \\
& \leqslant \sum_{n^{\prime} l^{\prime} m^{\prime}}\left|S_{n^{\prime} l^{\prime} m^{\prime}}^{n l m}(\mathbf{R})\right|^{2}=1 .
\end{aligned}
$$

We therefore find

$$
0 \leqslant\left|W_{n^{\prime} l^{\prime} m^{\prime}}^{n l m}(\mathbf{R})\right| \leqslant 1 .
$$

The function $W_{n^{\prime} l^{\prime} m^{\prime}}^{n l m}(\mathbf{R})$ can also be shown to have intimate relations with some familiar position-space quantities (see the Appendix). 


\section{FIRST ITERATED SOLUTIONS}

The momentum-space Schrödinger equation for a oneelectron diatomic system is given by ${ }^{4,5,8,9}$

$$
\begin{aligned}
\left(p^{2} / 2-E\right) \Psi(\mathbf{p})= & \left(2 \pi^{2}\right)^{-1} \int\left|\mathbf{p}-\mathbf{p}^{\prime}\right|^{-2} \\
& \times\left\{Z_{a} \exp \left[-i\left(\mathbf{p}-\mathbf{p}^{\prime}\right) \cdot \mathbf{R}_{a}\right]\right. \\
& \left.+Z_{b} \exp \left[-i\left(\mathbf{p}-\mathbf{p}^{\prime}\right) \cdot \mathbf{R}_{b}\right]\right\} \Psi\left(\mathbf{p}^{\prime}\right) d \mathbf{p}^{\prime}
\end{aligned}
$$

where $\mathbf{R}_{a}$ and $\mathbf{R}_{b}$ are position vectors of two nuclei whose charges are $Z_{a}$ and $Z_{b}$. The nuclear repulsion term has been omitted and $E$ represents the electronic energy. We discuss only the case of $E \leqslant 0$ and set

$$
p_{0}=\sqrt{-2 E} \text {. }
$$

We also assume that the momentum wave function is expressed as

$$
\Psi(\mathbf{p})=4 p_{0}^{5 / 2}\left(p^{2}+p_{0}^{2}\right)^{-2} \psi(\Omega)
$$

Then the Fock transformation [Eqs. (1) and (2)] allows us to rewrite Eq. (12a) as

$$
P(\Omega) \psi(\Omega)=p_{0} \psi(\Omega),
$$

where

$$
\begin{aligned}
P(\Omega) \psi(\Omega)= & \left(2 \pi^{2}\right)^{-1} \int\left[4 \sin ^{2}(\omega / 2)\right]^{-1} \\
& \times\left\{Z_{a} \exp \left[-i\left(\mathbf{p}-\mathbf{p}^{\prime}\right) \cdot \mathbf{R}_{a}\right]\right. \\
& \left.+Z_{b} \exp \left[-i\left(\mathbf{p}-\mathbf{p}^{\prime}\right) \cdot \mathbf{R}_{b}\right]\right\} \psi\left(\Omega^{\prime}\right) d \Omega^{\prime} \\
= & \sum_{n l m} n^{-1}\left\{Z_{a} \exp \left(-i \mathbf{p} \cdot \mathbf{R}_{a}\right) Y_{n l m}(\Omega)\right. \\
& \times \int\left[\exp \left(-i \mathbf{p}^{\prime} \cdot \mathbf{R}_{a}\right) Y_{n l m}\left(\Omega^{\prime}\right)\right]^{*} \psi\left(\Omega^{\prime}\right) d \Omega^{\prime} \\
& +Z_{b} \exp \left(-i \mathbf{p} \cdot \mathbf{R}_{b}\right) Y_{n l m}(\Omega) \\
& \left.\times \int\left[\exp \left(-i \mathbf{p}^{\prime} \cdot \mathbf{R}_{b}\right) Y_{n l m}\left(\Omega^{\prime}\right)\right]^{*} \psi\left(\Omega^{\prime}\right) d \Omega^{\prime}\right\}
\end{aligned}
$$

The first iterated solution $\psi^{(1)}$ for the initial guess $\psi^{(0)}$ (which may include some parameters to be determined) is defined by

$$
\psi^{(1)}(\Omega)=P(\Omega) \psi^{(0)}(\Omega) .
$$

In order to obtain approximate eigenvalue $p_{0}^{(1)}$ and wave function $\psi^{(1)}(\Omega)$ at the stage of the first iteration, we introduce a set of reference functions $\left\{\chi_{i}\right\}(i=1,2, \ldots, f)$ and consider the $\chi_{i}$ component (or the projection onto the $\chi_{i}$ axis in the functional space) of $\psi^{(0)}(\Omega)$ and $\psi^{(1)}(\Omega)$. From Eq. (12b), we then have

$p_{0}^{(1)}=\int \chi_{i}^{*}(\Omega) \psi^{(1)}(\Omega) d \Omega / \int \chi_{i}^{*}(\Omega) \psi^{(0)}(\Omega) d \Omega, \quad i=1,2, \ldots, f$

Equation (15) constitutes a set of equations from which we can determine $p_{0}^{(1)}$ and parameters embedded in $\psi^{(1)}(\Omega) . f$ is therefore governed by the number of unknown parameters. The reference functions $\left\{\chi_{i}\right\}$ are chosen by an appropriate physical insight on the system under consideration. Strictly speaking, there remains ambiguity for this choice, but we note that if $\psi^{(0)}(\Omega)$ is already a correct solution, then the result of Eq. (15) is independent of $\left\{\chi_{i}\right\}$.

\section{A. UA approximation}

As a starting function $\psi^{(0)}$, we may assume a hydrogenic $1 s$ function located at the origin of coordinates [see Eqs. (3e) and $(13 b)]$,

$$
\psi^{(0)}(\Omega)=Y_{100}(\Omega) .
$$

From Eqs. (12c) and (14), the first iterated solution is found to be

$$
\begin{aligned}
\psi^{(1)}(\Omega)= & \sum_{n l m}\left\{c_{n l m}^{a}\left[\exp \left(-i \mathbf{p} \cdot \mathbf{R}_{a}\right) Y_{n l m}(\Omega)\right]\right. \\
& \left.+c_{n l m}^{b}\left[\exp \left(-i \mathbf{p} \cdot \mathbf{R}_{b}\right) Y_{n l m}(\Omega)\right]\right\}
\end{aligned}
$$

where

$$
\begin{aligned}
& c_{n l m}^{a}=\left(Z_{a} / n\right) S_{n l m}^{100}\left(\mathbf{R}_{a}\right), \\
& c_{n l m}^{b}=\left(Z_{b} / n\right) S_{n l m}^{100}\left(\mathbf{R}_{b}\right) .
\end{aligned}
$$

Since $\left[\exp \left(-i \mathbf{p} \cdot \mathbf{R}_{a}\right) Y_{n l m}(\Omega)\right]$ represents a hydrogenic function $\Phi_{n l m}$ centered at $\mathbf{R}_{a}$, Eq. (17a) implies:

(i) $\psi^{(1)}$ is a mixing of an infinite number of hydrogenic functions centered at $\mathbf{R}_{a}$ and $\mathbf{R}_{b}$. Namely, the first iterated solution from the UA starting function is an LCAO function. It is interesting to observe that the concept of LCAO emerges in a purely mathematical manner without invoking any physical intuition.

(ii) Mixing coefficients are inversely proportional to the principal quantum number $n$. The ratio of mixing coefficients of the $(n, l, m)$ functions on different centers is

$$
c_{n l m}^{b} / c_{n l m}^{a}=\left(Z_{b} / Z_{a}\right)\left[S_{n l m}^{100}\left(\mathbf{R}_{b}\right) / S_{n l m}^{100}\left(\mathbf{R}_{a}\right)\right],
$$

which shows that this ratio is proportional to that of nuclear charges and is dependent on the coordinate system through the function $S_{n l m}^{100}$.

In the present UA approximation, $f=1$ and hence $\chi_{1}(\Omega)=Y_{100}(\Omega)$ seems to be a most natural choice. We then have

$$
p_{0}^{(1)}=Z_{a} W_{100}^{100}\left(\mathbf{R}_{a}\right)+Z_{b} W_{100}^{100}\left(\mathbf{R}_{b}\right)
$$

where

$$
W_{100}^{100}(\mathbf{R})=[1-(1+t) \exp (-2 t)] / t, \quad t=p_{0}^{(1)}|\mathbf{R}|,
$$

and $p_{0}^{(1)}$ depends on the coordinate system. Note that $p_{0}^{(1)}$ also enters into the right-hand side of Eq. (18a).

For a small internuclear separation $R$, Eq. (18a) can be explicitly solved as a function of $R$ :

$$
\begin{aligned}
p_{0}^{(1)}= & Z_{u}-(2 / 3)\left[Z_{a} k^{2}+Z_{b}(1-k)^{2}\right] Z_{u}^{2} R^{2} \\
& +(2 / 3)\left[Z_{a} k^{3}+Z_{b}(1-k)^{3}\right] Z_{u}^{3} R^{3} \\
& +O\left(R^{4}\right),
\end{aligned}
$$

where we have used 


$$
\begin{aligned}
& Z_{u}=Z_{a}+Z_{b}, \quad R=\left|\mathbf{R}_{b}-\mathbf{R}_{a}\right|, \\
& R_{a}=\left|\mathbf{R}_{a}\right|=k R, \quad R_{b}=\left|\mathbf{R}_{b}\right|=(1-k) R .
\end{aligned}
$$

When we employ the center-of-the-charges-of-the-nuclei (CCN) coordinates, $k=Z_{b} / Z_{u}$ and we obtain

$$
\begin{aligned}
p_{0}^{(1)}= & Z_{u}-(2 / 3) Z_{a} Z_{b} Z_{u} R^{2} \\
& +(2 / 3) Z_{a} Z_{b}\left(Z_{a}^{2}+Z_{b}^{2}\right) R^{3}+O\left(R^{4}\right), \\
E^{(1)}= & -\left[p_{0}^{(1)}\right]^{2} / 2 \\
= & -(1 / 2) Z_{u}^{2}+(2 / 3) Z_{a} Z_{b} Z_{u}^{2} R^{2} \\
& -(2 / 3) Z_{a} Z_{b} Z_{u}\left(Z_{a}^{2}+Z_{b}^{2}\right) R^{3}+O\left(R^{4}\right) .
\end{aligned}
$$

Since the correct short-range behavior is known to be ${ }^{10}$

$$
\begin{aligned}
E & =-(1 / 2) Z_{u}^{2}+(2 / 3) Z_{a} Z_{b} Z_{u} R^{2}-(2 / 3) Z_{a} Z_{b} Z_{u}^{3} R^{3} \\
& +(2 / 5) Z_{a} Z_{b}\left[1-(64 / 27)\left(Z_{a} Z_{b} / Z_{u}^{2}\right)\right] Z_{u}^{4} R^{4}+O\left(R^{5}\right),
\end{aligned}
$$

the first iterated energy $E^{(1)}$ is correct up to the order of $R^{2}$. However, this is not true for coordinate systems other than the $\mathrm{CCN}$ one, and the use of the $\mathrm{CCN}$ coordinates is suggested for the UA approximation.

On the other hand, we find for a very large $R$ that

$$
p_{0}^{(1)} \rightarrow 0 \text { and } E^{(1)} \rightarrow 0 \text { as } R \rightarrow \infty,
$$

if we use the $\mathrm{CCN}$ coordinates. This is obviously incorrect and the results of Eqs. (20b) and (21) mean that the simple UA approximation is valid only for a relatively small internuclear distance.

\section{B. LCAO approximation}

We now assume a simple (unnormalized) LCAO function as the starting function,

$\psi^{(0)}(\Omega)=\left[c_{a} \exp \left(-i \mathbf{p} \cdot \mathbf{R}_{a}\right)+c_{b} \exp \left(-i \mathbf{p} \cdot \mathbf{R}_{b}\right)\right] Y_{100}(\Omega)$,

which corresponds to $c_{a}(1 s)_{a}+c_{b}(1 s)_{b}$. Since the above LCAO function is the initial and predominant term of the first iterated solution of the UA approximation [see Eq. (17a)], we expect a considerable improvement of the result.

Inserting Eq. (22) into Eq. (14), we obtain the first iterated function

$$
\begin{aligned}
\psi^{(1)}(\Omega)= & {\left[Z_{a} c_{a} \exp \left(-i \mathbf{p} \cdot \mathbf{R}_{a}\right)\right.} \\
& \left.+Z_{b} c_{b} \exp \left(-i \mathbf{p} \cdot \mathbf{R}_{b}\right)\right] Y_{100}(\Omega) \\
& +\sum_{n l m} n^{-1}\left[Z_{a} c_{b} S_{n l m}^{100}(-\mathbf{R}) \exp \left(-i \mathbf{p} \cdot \mathbf{R}_{a}\right)\right. \\
& \left.+Z_{b} c_{a} S_{n l m}^{100}(\mathbf{R}) \exp \left(-i \mathbf{p} \cdot \mathbf{R}_{b}\right)\right] Y_{n l m}(\Omega)
\end{aligned}
$$

which is again an LCAO function composed of various hydrogenic functions centered at $\mathbf{R}_{a}$ and $\mathbf{R}_{b}$.

Since the ratio $c_{b} / c_{a}$ is meaningful, $f=2$ for the present LCAO approximation. For the two reference functions, we choose $\chi_{1}=\exp \left(-i \mathbf{p} \cdot \mathbf{R}_{a}\right) Y_{100}(\Omega)$ and $\chi_{2}$ $=\exp \left(-i \mathbf{p} \cdot \mathbf{R}_{b}\right) Y_{100}(\Omega)$ which correspond to $(1 s)_{a}$ and $(1 s)_{b}$, respectively. The projections of $\psi^{(0)}$ and $\psi^{(1)}$ onto these reference functions are found to be
$(1 s)_{a}$ component:

$\int \chi_{1}^{*} \psi^{(0)} d \Omega=c_{a}+\left[S_{100}^{100}(\mathbf{R})\right]^{*} c_{b}$,

$\int \chi_{1}^{*} \psi^{(1)} d \Omega=\left[Z_{a}+Z_{b} W_{100}^{100}(\mathbf{R})\right] c_{a}+Z_{u}\left[S_{100}^{100}(\mathbf{R})\right]^{*} c_{b}$

$(1 s)_{b}$ component:

$$
\begin{aligned}
\int \chi_{2}^{*} \psi^{(0)} d \Omega= & {\left[S_{100}^{100}(\mathbf{R})\right] c_{a}+c_{b}, } \\
\int \chi_{2}^{*} \psi^{(1)} d \Omega= & Z_{u}\left[S_{100}^{100}(\mathbf{R})\right] c_{a} \\
& +\left\{Z_{a}\left[W_{100}^{100}(\mathbf{R})\right]^{*}+Z_{b}\right\} c_{b},
\end{aligned}
$$

where $\mathbf{R}=\mathbf{R}_{b}-\mathbf{R}_{a}, W_{100}^{100}(\mathbf{R})$ is given in Eq. $(18 \mathrm{~b})$, and

$$
S_{100}^{100}(\mathbf{R})=(1+t) \exp (-t), \quad t=p_{0}^{(1)}|\mathbf{R}| .
$$

From Eq. (15), we then have the following equation for the determination of $p_{0}^{(1)}$ and $c_{b} / c_{a}$ :

$$
\begin{aligned}
& \left(\begin{array}{cc}
p_{0}^{(1)}-Z_{a}-Z_{b} W_{100}^{100} & \left(p_{0}^{(1)}-Z_{u}\right)\left[S_{100}^{100}\right]^{*} \\
\left(p_{0}^{(1)}-Z_{u}\right) S_{100}^{100} & p_{0}^{(1)}-Z_{a}\left[W_{100}^{100}\right]^{*}-Z_{b}
\end{array}\right)\left(\begin{array}{l}
c_{a} \\
c_{b}
\end{array}\right) \\
& \quad=\left(\begin{array}{l}
0 \\
0
\end{array}\right) .
\end{aligned}
$$

Note that Eq. (25) is linear with respect to $c_{a}$ and $c_{b}$ but is nonlinear with respect to $p_{0}^{(1)}$ because of the functions $\boldsymbol{W}_{100}^{100}$ and $S_{100}^{100}$. Different from the UA approximation, $p_{0}^{(1)}$ is independent of the coordinate system employed.

As in the UA approximation, the explicit solution of Eq. (25) is possible for a small internuclear separation $R$. The results for $p_{0}^{(1)}$ and $E^{(1)}$ are

$$
\begin{aligned}
p_{0}^{(1)}= & Z_{u}-(2 / 3) Z_{a} Z_{b} Z_{u} R^{2}+(2 / 3) Z_{a} Z_{b} Z_{u}^{2} R^{3} \\
& -(2 / 5) Z_{a} Z_{b}\left[1-(5 / 9) Z_{a} Z_{b} / Z_{u}^{2}\right] Z_{u}^{3} R^{4}+O\left(R^{5}\right)
\end{aligned}
$$

$$
\begin{aligned}
E^{(1)}= & -(1 / 2) Z_{u}^{2}+(2 / 3) Z_{a} Z_{b} Z_{u}^{2} R^{2}-(2 / 3) Z_{a} Z_{b} Z_{u}^{3} R^{3} \\
& +(2 / 5) Z_{a} Z_{b}\left[1-(10 / 9) Z_{a} Z_{b} / Z_{u}^{2}\right] Z_{u}^{4} R^{4} \\
& +O\left(R^{5}\right) .
\end{aligned}
$$

$E^{(1)}$ agrees completely with the correct result [Eq. (20c)] up to the order of $R^{3}$. The coefficient of $R^{4}$ in $E^{(1)}$ is partly correct. The asymptotic behavior for a very large $R$ is

$$
\left.\begin{array}{l}
p_{0}^{(1)} \rightarrow\left[Z_{u}+\left|Z_{a}-Z_{b}\right|\right] / 2 \\
E^{(1)} \rightarrow-\left[Z_{u}+\left|Z_{a}-Z_{b}\right|\right]^{2} / 8
\end{array}\right\} \text { as } R \rightarrow \infty,
$$

and correctly converges to the atom with larger nuclear charge. As expected, the LCAO approximation has good asymptotic behaviors both at $R \rightarrow 0$ and $R \rightarrow \infty$, and supports the adequacy of the choice of the reference functions $(1 s)_{a}$ and $(1 s)_{b}$. However, numerical analysis for some specific systems is required to see the validity in the intermediate range of $R$.

\section{Numerical results for $\mathrm{H}_{2}^{+}$and $\mathrm{HeH}^{2+}$}

We have calculated the first iterated energy $E^{(1)}$ based on Eq. (18a) (UA approximation with the CCN coordinates) 
TABLE I. Negatives of electronic energies (a.u.) for the ground state of the $\mathrm{H}_{2}^{+}$system. Values in parentheses are percent errors.

\begin{tabular}{ccccc}
\hline \hline & \multicolumn{2}{c}{ First iterated } & Variational & Exact \\
& LCAO & \\
\cline { 2 - 3 } & UA & LCAO & & \\
\hline 0.0 & $2.00000(0.0)$ & $2.00000(0.0)$ & $2.00000(0.0)$ & 2.00000 \\
0.1 & $1.97619(0.1)$ & $1.97817(0.0)$ & $1.97821(0.0)$ & 1.97824 \\
0.2 & $1.91653(0.6)$ & $1.92799(0.0)$ & $1.92833(0.0)$ & 1.92862 \\
0.3 & $1.83676(1.6)$ & $1.86481(0.1)$ & $1.86579(0.0)$ & 1.86670 \\
0.4 & $1.74826(2.9)$ & $1.79693(0.2)$ & $1.79884(0.1)$ & 1.80075 \\
0.5 & $1.65812(4.4)$ & $1.72867(0.4)$ & $1.73176(0.2)$ & 1.73499 \\
0.6 & $1.57036(6.0)$ & $1.66225(0.6)$ & $1.66675(0.3)$ & 1.67148 \\
0.8 & $1.40908(9.4)$ & $1.53872(1.0)$ & $1.54657(0.5)$ & 1.55448 \\
1.0 & $1.26982(12.5)$ & $1.42934(1.5)$ & $1.44100(0.7)$ & 1.45179 \\
1.5 & $1.00540(19.5)$ & $1.21284(2.9)$ & $1.23379(1.2)$ & 1.24899 \\
2.0 & $0.82498(25.2)$ & $1.05918(3.9)$ & $1.08651(1.5)$ & 1.10263 \\
2.5 & $0.69640(29.9)$ & $0.94869(4.5)$ & $0.97876(1.5)$ & 0.99382 \\
3.0 & $0.60091(34.0)$ & $0.86787(4.7)$ & $0.89778(1.4)$ & 0.91090 \\
4.0 & $0.46955(41.0)$ & $0.76250(4.2)$ & $0.78733(1.1)$ & 0.79608 \\
5.0 & $0.38405(47.0)$ & $0.70100(3.2)$ & $0.71921(0.7)$ & 0.72442 \\
6.0 & $0.32426(52.2)$ & $0.66292(2.3)$ & $0.67575(0.4)$ & 0.67864 \\
8.0 & $0.24654(60.7)$ & $0.61989(1.2)$ & $0.62673(0.1)$ & 0.62757 \\
10.0 & $0.19848(67.0)$ & $0.59600(0.8)$ & $0.60030(0.0)$ & 0.60058 \\
15.0 & $0.13305(76.5)$ & $0.56470(0.4)$ & $0.56667(0.0)$ & 0.56672 \\
20.0 & $0.09993(81.8)$ & $0.54886(0.2)$ & $0.55000(0.0)$ & 0.55001 \\
& & & & \\
\hline \hline
\end{tabular}

${ }^{a}$ References 11 and 12 .

and Eq. (25) (LCAO approximation), and compared the results with the corresponding variational LCAO and exact values for a wide range of $R$. The results are summarized in Tables I and II for the $\mathrm{H}_{2}^{+}$and $\mathrm{HeH}^{2+}$ systems, respectively.

In both systems, the general feature of the UA and LCAO approximations is consistent with the analysis given in the previous subsections. The UA approximation is valid only for very small values of $R$. The error increases monotonically as $R$ increases. On the other hand, the results of the

TABLE II. Negatives of electronic energies (a.u.) for the ground state of the $\mathrm{HeH}^{2+}$ system. Values in parentheses are percent errors.

\begin{tabular}{|c|c|c|c|c|}
\hline \multirow{2}{*}{$R$} & \multicolumn{2}{|c|}{ First iterated } & \multirow{2}{*}{$\begin{array}{c}\text { Variational } \\
\text { LCAO }\end{array}$} & \multirow{2}{*}{ Exact $^{a}$} \\
\hline & UA & LCAO & & \\
\hline 0.0 & $4.50000(0.0)$ & $4.50000(0.0)$ & $4.50000(0.0)$ & 4.50000 \\
\hline 0.1 & $4.40064(0.2)$ & $4.41083(0.0)$ & $4.41110(0.0)$ & 4.41132 \\
\hline 0.2 & $4.17792(1.3)$ & $4.22934(0.1)$ & $4.23107(0.0)$ & 4.23270 \\
\hline 0.3 & 3.91289 ( 3.0$)$ & $4.02532(0.2)$ & $4.02967(0.1)$ & 4.03414 \\
\hline 0.4 & $3.64678(5.1)$ & $3.82602(0.4)$ & $3.83388(0.2)$ & 3.84206 \\
\hline 0.5 & $3.39690(7.3)$ & $3.64153(0.7)$ & $3.65354(0.3)$ & 3.66555 \\
\hline 0.6 & $3.16899(9.6)$ & $3.47504(0.9)$ & $3.49153(0.4)$ & 3.50689 \\
\hline 0.8 & $2.77946(14.2)$ & $3.19626(1.4)$ & $3.22127(0.6)$ & 3.24084 \\
\hline 1.0 & $2.46584(18.7)$ & $2.98194(1.7)$ & $3.01296(0.7)$ & 3.03336 \\
\hline 1.5 & $1.91034(29.1)$ & $2.64894(1.7)$ & $2.68088(0.5)$ & 2.69546 \\
\hline 2.0 & $1.55224(38.2)$ & $2.48064(1.3)$ & $2.50435(0.3)$ & 2.51220 \\
\hline 2.5 & $1.30415(45.8)$ & $2.38441(0.9)$ & $2.40100(0.2)$ & 2.40489 \\
\hline 3.0 & $1.12274(51.9)$ & $2.32159(0.6)$ & $2.33354(0.1)$ & 2.33549 \\
\hline 4.0 & $0.87598(61.1)$ & $2.24304(0.3)$ & $2.25001(0.0)$ & 2.25061 \\
\hline 5.0 & $0.71651(67.4)$ & $2.19545(0.2)$ & $2.20000(0.0)$ & 2.20024 \\
\hline 6.0 & $0.60527(72.1)$ & $2.16346(0.2)$ & $2.16667(0.0)$ & 2.16678 \\
\hline 8.0 & $0.46075(78.3)$ & $2.12316(0.1)$ & $2.12500(0.0)$ & 2.12504 \\
\hline 10.0 & $0.37128(82.3)$ & $2.09881(0.1)$ & $2.10000(0.0)$ & 2.10002 \\
\hline 15.0 & $0.24922(87.9)$ & $2.06613(0.0)$ & $2.06667(0.0)$ & 2.06667 \\
\hline 20.0 & $0.18728(90.9)$ & $2.04970(0.0)$ & $2.05000(0.0)$ & 2.05000 \\
\hline
\end{tabular}

${ }^{\mathrm{a}}$ References 13 and 14 .
LCAO approximation are acceptable not only for the small and large $R$ regions but also for the intermediate region. The maximum errors relative to the exact values are found to be $4.7 \%$ at $R=3.0\left(\mathrm{H}_{2}^{+}\right)$and $1.7 \%$ at $R=1.0-1.5\left(\mathrm{HeH}^{2+}\right)$. The LCAO approximation is reliable semiquantitatively, and the iterative solution with this method seems to be promising.

When compared with the corresponding variational LCAO calculation, however, the error of the first iterated LCAO is about three times larger in the intermediate $R$ region, and the first iterated LCAO solution is still open to further improvement. Second and more iterations are possible methods for this purpose. Another direction of improvement is the use of an extended LCAO starting function which includes some additional AO's such as $2 p \sigma \mathrm{AO}$.

However, the primary merit of the momentum space approach will not be to obtain accurate numerical values, since the usual variational method and its sophisticated version in position space are obviously more direct and powerful for this purpose as long as the problem of electronic structures of atoms and molecules is concerned. Rather, the momentum space approach can be regarded as a hopeful tool which permits us to explore new (or complementary, at least) concepts and pictures that are "hidden" in the ordinary position space but useful to grope for a new development in the electronic structure theory. Nonvariational calculation and the resultant analysis of short-range interactions, presented in this study, may be located along this line, in which we have seen that fewer kinds of integrals are sufficient than those required in the variational calculations.

\section{ACKNOWLEDGMENT}

Part of this study has been supported by a Grant-in-Aid for Scientific Research from the Ministry of Education of Japan.

\section{APPENDIX}

The following position-space interpretation is possible for the functions $S_{n^{\prime} l^{\prime} m^{\prime}}^{n l m}(\mathbf{R})$ and $W_{n^{\prime} l^{\prime} m^{\prime}}^{n l m}(\mathbf{R})$ introduced in Sec. II.

Let $u_{n l m}(\mathbf{r})$ be the hydrogenic wave function in position space. Then the following correspondence holds through the Fourier transformation (FT):

$$
\begin{aligned}
& u_{n l m}(\mathbf{r}-\mathbf{R}) \stackrel{\text { FT }}{\leftrightarrow} \exp (-i \mathbf{p} \cdot \mathbf{R}) \Phi_{n l m}(\mathbf{p}), \\
& |\mathbf{r}|^{-1} u_{n l m}(\mathbf{r}) \stackrel{\text { FT }}{\leftrightarrow}\left(2 \pi^{2}\right)^{-1} \int\left|\mathbf{p}-\mathbf{p}^{\prime}\right|^{-2} \Phi_{n l m}\left(\mathbf{p}^{\prime}\right) d \mathbf{p}^{\prime} \\
& =\left[2 p_{0}^{3 / 2} /\left(p^{2}+p_{0}^{2}\right)\right] Y_{n l m}(\Omega) .
\end{aligned}
$$

Therefore we obtain

$$
\begin{aligned}
\int u_{n^{\prime} l^{\prime} m^{\prime}}^{*}(\mathbf{r}-\mathbf{R})|\mathbf{r}|^{-1} u_{n l m}(\mathbf{r}) d \mathbf{r} \\
=\int\left[\exp (-i \mathbf{p} \cdot \mathbf{R}) \Phi_{n^{\prime} l^{\prime} m^{\prime}}(\mathbf{p})\right]^{*} \\
\quad \times\left[2 p_{0}^{3 / 2} /\left(p^{2}+p_{0}^{2}\right)\right] Y_{n l m}(\Omega) d \mathbf{p}
\end{aligned}
$$




$$
\begin{aligned}
& =p_{0} \int \exp (i \mathbf{p} \cdot \mathbf{R}) Y_{n^{\prime} l^{\prime} m^{\prime}}^{*}(\Omega) Y_{n l m}(\Omega) d \Omega \\
& =p_{0} S_{n^{\prime} l^{\prime} m^{\prime}}^{n l m}(\mathbf{R}),
\end{aligned}
$$

which implies that the function $S_{n^{\prime} l^{\prime} m^{\prime}}^{n l m}(\mathbf{R})$ is proportional to the nuclear attraction integral in position space over the hydrogenic functions. As a result,

$$
S_{n^{\prime} l^{\prime} m^{\prime}}^{n l m}(\mathbf{R}) \rightarrow 0 \text { as }|\mathbf{R}| \rightarrow \infty .
$$

Alternative correspondence was given by Shibuya and Wulf$\operatorname{man}^{4}$ (see also Refs. 5 and 9). The result is

$$
\begin{aligned}
S_{n^{\prime} l^{\prime} m^{\prime}}^{n l m}(\mathbf{R})= & (1 / 2) \sum_{v \lambda \mu}\left(2 \pi / p_{0}\right)^{3 / 2} V_{n^{\prime} l^{\prime} m^{\prime}, v \lambda \mu}^{n l m} u_{v \lambda \mu}(\mathbf{R}), \\
V_{n^{\prime} l^{\prime} m^{\prime}, v \lambda \mu}^{n l m}= & \left(2 \pi^{2}\right)^{1 / 2} \int\left[2 Y_{100}(\Omega)+Y_{200}(\Omega)\right] Y_{n l m}(\Omega) \\
& \times Y_{n^{\prime} l^{\prime} m^{\prime}}^{*}(\Omega) Y_{v \lambda \mu}^{*}(\Omega) d \Omega,
\end{aligned}
$$

and $S_{n^{\prime} l^{\prime} m^{\prime}}^{n l m}(\mathbf{R})$ is expressible by a linear combination of a finite number of position-space hydrogenic functions.

Similarly, considering another correspondence

$$
\begin{aligned}
|\mathbf{r}-\mathbf{R}|^{-1} u_{n l m}(r) \leftrightarrow( & \left(2 \pi^{2}\right)^{-1} \int\left|\mathbf{p}-\mathbf{p}^{\prime}\right|^{-2} \exp \left[-i\left(\mathbf{p}-\mathbf{p}^{\prime}\right)\right. \\
& \cdot \mathbf{R}] \Phi_{n l m}\left(\mathbf{p}^{\prime}\right) d \mathbf{p}^{\prime} \\
= & {\left[2 p_{0}^{3 / 2} /\left(p^{2}+p_{0}^{2}\right)\right] \exp (-i \mathbf{p} \cdot \mathbf{R}) . } \\
& \times \sum_{v \lambda \mu} v^{-1} S_{v \lambda \mu}^{n l m}(\mathbf{R}) Y_{v \lambda \mu}(\Omega)
\end{aligned}
$$

we have

$$
\int u_{n^{\prime} l^{\prime} m^{\prime}}^{*}(\mathbf{r})|\mathbf{r}-\mathbf{R}|^{-1} u_{n l m}(\mathbf{r}) d \mathbf{r}
$$

$$
\begin{aligned}
= & p_{0} \int\left[\sum_{v \lambda \mu} v^{-1} S_{v \lambda \mu}^{n l m}(\mathbf{R}) Y_{v \lambda \mu}(\Omega)\right] \\
& \times\left[\exp (i \mathbf{p} \cdot \mathbf{R}) Y_{n^{\prime} l^{\prime} m^{\prime}}(\Omega)\right]^{*} d \Omega \\
= & p_{0} W_{n^{\prime} l^{\prime} m^{\prime}}^{n l m}(\mathbf{R}) .
\end{aligned}
$$

Thus the function $W_{n^{\prime} l^{\prime} m^{\prime}}^{n l m}(\mathbf{R})$ is proportional to another kind of nuclear attraction integral in position space, and

$$
W_{n^{\prime} l^{\prime} m^{\prime}}^{n l m}(\mathbf{R}) \rightarrow 0 \text { as }|\mathbf{R}| \rightarrow \infty
$$

We note that in the above equations, all the exponents of $\left\{u_{n l m}(\mathbf{r})\right\}$ should be taken to be $p_{0}$.

${ }^{1}$ T. Koga, J. Chem. Phys. 82, 2022 (1985).

${ }^{2}$ T. Koga and S. Matsumoto, J. Chem. Phys. 82, 5127 (1985).

${ }^{3}$ V. Fock, Z. Phys. 98, 145 (1935).

${ }^{4}$ T. Shibuya and C. E. Wulfman, Proc. R. Soc. London, Ser. A 286, 376 (1965); Am. J. Phys. 33, 570 (1965); C. E. Wulfman, in Group Theory and Its Applications, edited by E. M. Loeble (Academic, New York, 1971), Vol. 2.

${ }^{5}$ B. R. Judd, Angular Momentum Theory for Diatomic Molecules (Academic, New York, 1975).

${ }^{6}$ L. C. Biedenharn and J. D. Louck, Angular Momentum in Quantum Physics. Theory and Application (Addison-Wesley, Reading, Mass., 1981).

${ }^{7}$ M. I. Petrashen and E. D. Trifonov, Applications of Group Theory in Quantum Mechanics (MIT, Cambridge, 1969).

${ }^{8}$ R. McWeeny, Proc. Phys. Soc. A 62, 519 (1949).

${ }^{9}$ H. J. Monkhorst and B. Jeriorski, J. Chem. Phys. 71, 5268 (1979).

${ }^{10}$ W. Byers Brown and E. Steiner, J. Chem. Phys. 44, 3934 (1966).

${ }^{11}$ D. R. Bates, K. Ledsham, and A. L. Stewart, Philos. Trans. R. Soc. London Sect. A 246, 215 (1954).

${ }^{12}$ H. Wind, J. Chem. Phys. 42, 2371 (1965).

${ }^{13}$ D. R. Bates and T. R. Carson, Proc. R. Soc. London Ser. A 234, 207 (1956).

${ }^{14}$ T. G. Winter, M. D. Duncan, and N. F. Lane, J. Phys. B 10, 285 (1977). 